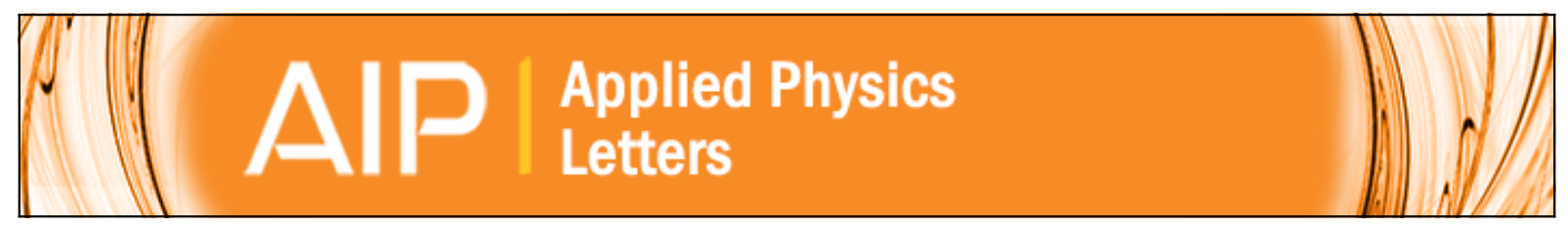

\title{
Spin-wave interference
}

Sangkook Choi, Ki-Suk Lee, and Sang-Koog Kim

Citation: Applied Physics Letters 89, 062501 (2006); doi: 10.1063/1.2259813

View online: http://dx.doi.org/10.1063/1.2259813

View Table of Contents: http://scitation.aip.org/content/aip/journal/apl/89/6?ver=pdfcov

Published by the AIP Publishing

\section{Articles you may be interested in}

Magnetostatic spin-wave modes of an in-plane magnetized garnet-film disk

J. Appl. Phys. 113, 103901 (2013); 10.1063/1.4794318

Engineering spin-wave channels in submicrometer magnonic waveguides

AIP Advances 3, 032144 (2013); 10.1063/1.4799738

Micromagnetic study of spin wave propagation in bicomponent magnonic crystal waveguides

Appl. Phys. Lett. 98, 153107 (2011); 10.1063/1.3579531

Anisotropic spin-wave patterns generated by spin-torque nano-oscillators

J. Appl. Phys. 109, 07 C733 (2011); 10.1063/1.3566000

Double-contact spin-torque nano-oscillator with optimized spin-wave coupling: Micromagnetic modeling Appl. Phys. Lett. 90, 083114 (2007); 10.1063/1.2696256

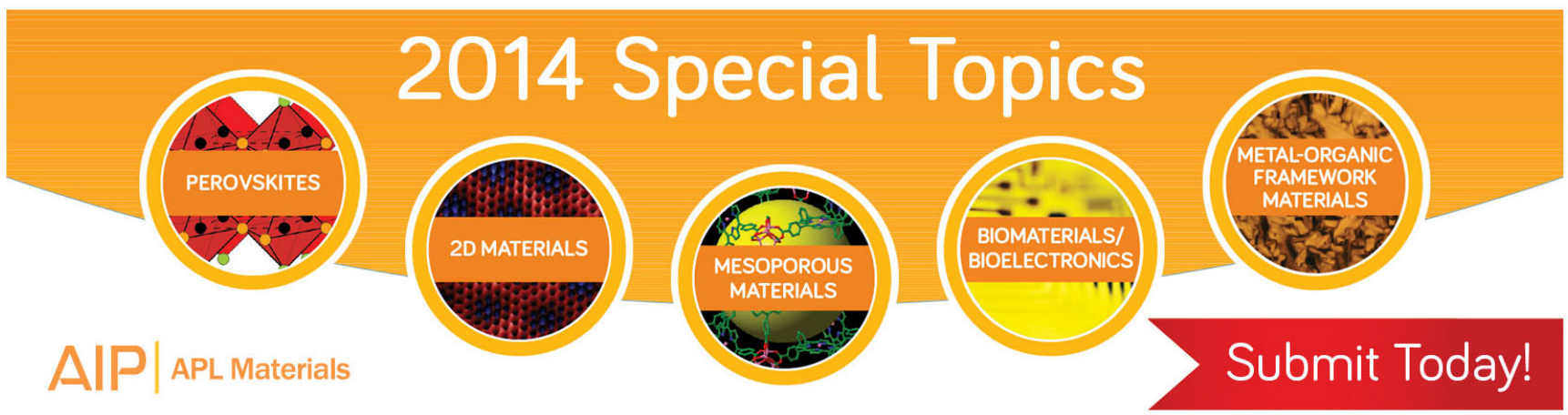




\title{
Spin-wave interference
}

\author{
Sangkook Choi, Ki-Suk Lee, and Sang-Koog Kim ${ }^{\text {a) }}$ \\ Research Center for Spin Dynamics and Spin-Wave Devices, Seoul National University, Seoul 151-744, \\ South Korea and Nanospintronics Laboratory, School of Materials Science and Engineering, \\ College of Engineering, Seoul National University, Seoul 151-744, South Korea
}

(Received 24 February 2006; accepted 8 June 2006; published online 7 August 2006)

\begin{abstract}
Spin-wave interference is demonstrated in the micromagnetic modeling of a specially designed geometry made of variously shaped magnetic thin-film waveguides. When spin waves are diffracted through two separate openings, corresponding to the two pinholes in the second screen of Young's apparatus, they interfere constructively or destructively in a magnetic medium, thereby showing distinct interference patterns. Furthermore, the radiation, propagation, transmission, and dispersion behaviors of spin waves as well as the filtering of their lower frequencies are investigated in the present modeling study. These results directly confirm not only the wave characteristics of spin waves traveling at ultrafast speeds in variously shaped magnetic waveguides but also their interference effect, that is similar to that observed in well-known Young's double slit experiment with light. (C) 2006 American Institute of Physics. [DOI: 10.1063/1.2259813]
\end{abstract}

Recently, a radiation behavior of spin waves (SWs), which is driven by the motion of a magnetic vortex core and the annihilation of a magnetic vortex-antivortex pair, was found in a confined ferromagnetic thin-film model system. ${ }^{1}$ From a technological point of view, such a radiation behavior and SW propagations at ultrafast speeds through variously shaped magnetic waveguides (e.g., a nanowire) have the potential to be used for logical operations in one of the next generations of logic devices. ${ }^{2}$ In this letter, we report on SW interference obtained from micromagnetic simulations conducted in a specially designed geometry that imitates Young's double slit experiment. ${ }^{3}$ Moreover, the wave characteristics of the SWs, such as their radiation, propagation, reflection, transmission, dispersion, and the filtering of lower frequencies are reported.

The geometry of the model system used in the present study is made of a $10 \mathrm{~nm}$ thick $\mathrm{Ni}_{80} \mathrm{Fe}_{20}$ (Permalloy: Py) magnetic film and is composed of three different components: a circular shaped disk, a Y-shaped nanowire waveguide incorporating a $100 \mathrm{~nm}$ long Fe segment (placed between $x=200$ and $300 \mathrm{~nm}$ ) in its single channel part, and a $\Xi$-shaped magnetic medium, as shown in Fig. 1. In the present micromagnetic simulations, ${ }^{4}$ we used a constant cell size of $2.5 \times 2.5 \times 10 \mathrm{~nm}^{3}$ and a damping parameter of $\alpha$ $=0.01$. For the Py material, we used an exchange stiffness constant of $A=1.3 \times 10^{-11} \mathrm{~J} / \mathrm{m}$ and a saturation magnetization of $M_{s}=8.6 \times 10^{5} \mathrm{~A} / \mathrm{m}$. For the Fe segment, we used $A$ $=2.1 \times 10^{-11} \mathrm{~J} / \mathrm{m}$ and $M_{s}=1.7 \times 10^{6} \mathrm{~A} / \mathrm{m}$. For the value of $A$ at the interface between the different materials, viz., Fe and Py, their harmonic mean of $1.6 \times 10^{-11} \mathrm{~J} / \mathrm{m}$ was used. For both materials, the magnetic anisotropy constant is assumed to be of $K=0$ just for such a model study. The geometry shown in Fig. 1 is specially designed to reproduce certain interference patterns of SWs, which are similar to those observed in Young's double slit experiment. In the model geometry, a magnetic vortex in the circular disk is thought to play a crucial role in the production of SWs by acting as a radiation source, while the Y-shaped waveguide allows the

a) Author to whom correspondence should be addressed; electronic mail: radiation-mode $\mathrm{SWs}$ to be injected into and propagate through it. The $\Xi$-shaped geometry plays the role of a magnetic medium wherein the SWs being diffracted from the two openings marked by $P_{2}$ propagate through and interfere.

An initial magnetization configuration at equilibrium in the given geometry under zero magnetic field is shown in Fig. 1, as indicated by the colors of the local areas. The circular disk has its own magnetic vortex state, the Y-shaped waveguide is saturated along its long channel, and the $\Xi$-shaped medium is almost uniformly magnetized along the orientation as indicated by the red color, as designed. ${ }^{5}$ The initial spin configuration is then intentionally perturbed using a sinusoidal magnetic field pulse with an amplitude of $H$ $=300 \mathrm{Oe}$ and a period of $\Delta t=0.2 \mathrm{~ns}$ (i.e., $5 \mathrm{GHz}$ ), applied only to the circular disk along the $+y$ direction. Upon applying this one period pulse, SWs start to radiate from the vortex core, as visualized by the series of snapshot images taken at the indicated times shown in Fig. 2(a). ${ }^{6}$ These SWs have large amplitudes and exist in a definite form of optical radiation waves. The details and underlying physics will be reported elsewhere.

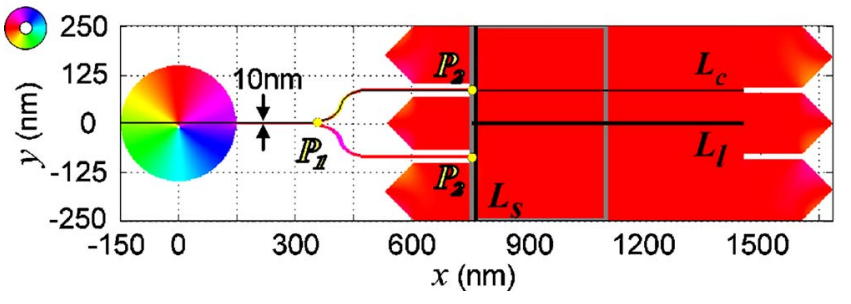

FIG. 1. (Color online) Model geometry of a $10 \mathrm{~nm}$ thick Py film incorporating an Fe film at position of $200<x<300 \mathrm{~nm}$ and its lateral dimensions along with the coordinates used in the micromagnetic simulation of spinwave interference. The colors represent the in-plane orientations of the local magnetizations at equilibrium in the given geometry under zero magnetic field, as indicated by the colored wheel in the top left-hand corner. The point marked by $P_{1}$ is situated at $(x, y)=(360,0)$; the two points marked by $P_{2}$ are at $(755,85)$ and $(755,-85)$. The gray-colored rectangular box indicates the area of $755<x<1100$ and $-250<y<250$. The straight lines of $L_{l}$ and $L_{s}$ are placed at $755<x<1455$ and $y=0$, and $x=765$ and $-250<y<250$, respectively, while the curved line of $L_{c}$ is situated along the black-colored line, as noted, in the region of $-150<x<1455$ and $0<y<85$. 
(a)

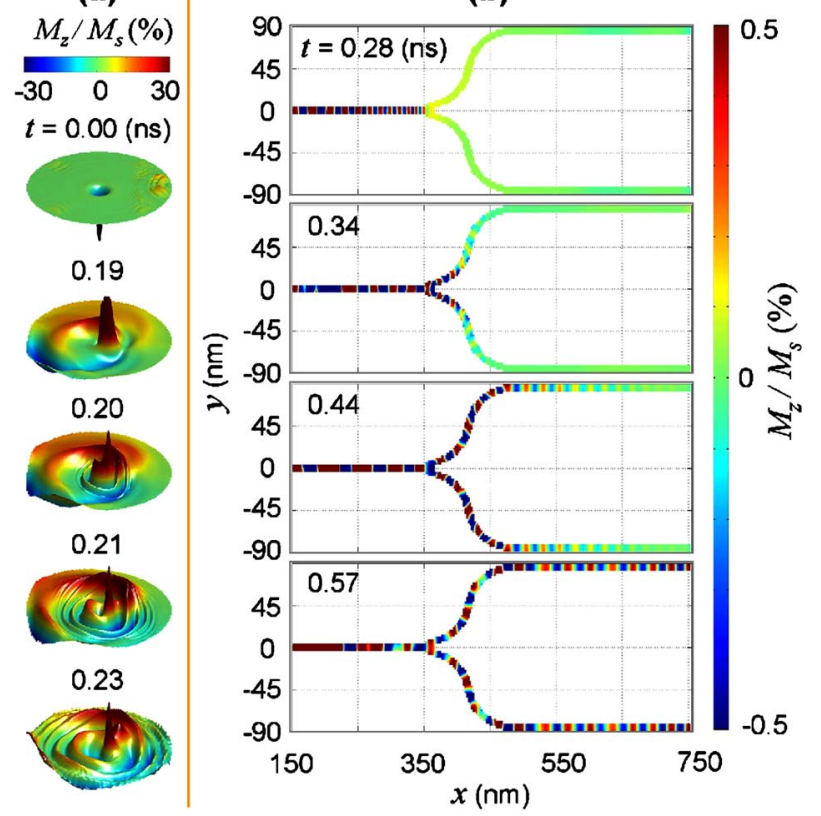

FIG. 2. (Color online) Perspective-view images of the spatial distributions of the local $M_{z} / M_{s}$ components for the circular disk in (a) and plane view images for the Y-shaped waveguide in (b) at the indicated times. The color bars indicate the $M_{z} / M_{s}$ components.

The propagation behavior of the radiation-mode SWs through the Y-shaped waveguide, after being injected into it from the vortex state, is also well described in Fig. 2(b). ${ }^{6}$ The distributions of the out-of-plane components of the local magnetizations normalized by their saturation values, $M_{z} / M_{s}$, indicate that the SWs being propagated along the single channel are then separated into the two independent paths of the branched channel. The openings marked by $P_{1}$ and $P_{2}$ in the Y-shaped waveguide act as the single pinhole and the two pinholes in the first and second screens, respectively, of Young's double slit experiment. When arriving at the end $P_{2}$ of the two independent paths, the SWs diffract coherently into the $\Xi$-shaped medium, because they have almost the same phase at $P_{2}$.

Next, an interesting phenomenon occuring in the $\Xi$-shaped medium is that the wave fronts of the SWs flare out into it in all of the in-plane directions and then interfere with each other, as shown in Fig. 3(a). ${ }^{6}$ The SW interference pattern is clearly seen by displaying a spatial configuration of the $M_{z} / M_{s}$ components taken at $t=0.67$ ns only in the area marked by the gray-colored rectangular box shown in Fig. 1. The SWs diffracted from $P_{2}$, indicated by the two small points, are superimposed on each other at every position in the $\Xi$-shaped medium and interfere constructively or destructively, thereby making the distinct interference pattern. This pattern is also examined by conducting fast-Fouriertransform (FFT) analyses. ${ }^{7,8}$ The amplitude and phase distributions of the FFT of the local $M_{z} / M_{s}$ components represent the pattern of the nodes and antinodes of the SWs, shown in Figs. 3(b) and 3(c), respectively, for a specific frequency of $f=36.5 \mathrm{GHz}$, along with their attenuations as they propagate far away from $P_{2}$. Moreover, their interference characteristics provide clear evidence for the superposition principle.

Figure 4 shows the $f$ spectra as a function of the distance of propagation of the SWs along the line indicated by $L_{c}$ in Fig. 1. As shown in Fig. 4(a), the radiation modes in the
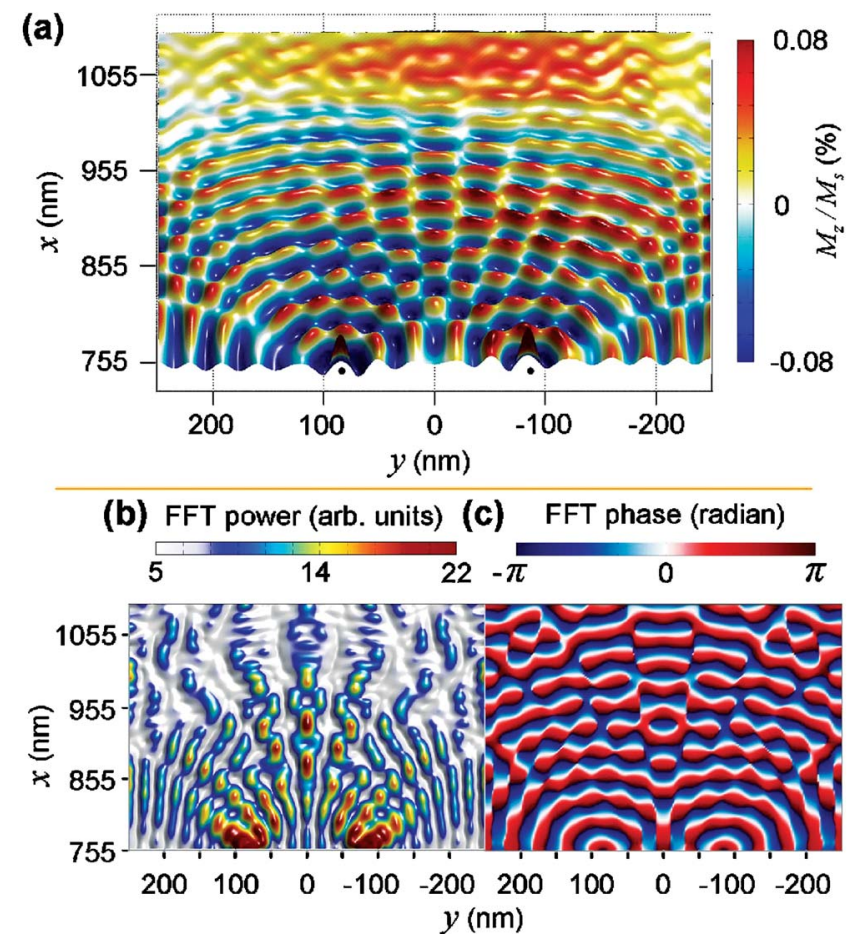

FIG. 3. (Color online) (a) Snapshot image taken at $t=0.67 \mathrm{~ns}$ for the dynamic evolution of the local $M_{z} / M_{s}$ distribution in the area marked by the gray-colored rectangular box shown in Fig. 1, illustrating the interference pattern of SWs diffracted through the two openings marked by $P_{2}$. (b) and (c) show their FFT power and phase for $f=36.5 \mathrm{GHz}$, respectively.

finite-sized circular disk have various radial nodes depending on $f$, due to their quantized wavelengths $\lambda$ able to exist in the given diameter. Regarding the Y-shaped waveguide, the $f$ spectra in Fig. 4(b) exhibit contrasting features between the characteristic individual regions, including a single channel $(\alpha, \beta$, and $\gamma)$, a curved channel $(\delta)$, and a branched channel $(\varepsilon)$. Among all the radiation modes, those SWs with approximate values of $f>13 \mathrm{GHz}$ are injected into the $\alpha$ region made of Py placed before the Fe $\beta$ region. Upon being injected into the Fe $\beta$ region, those SWs whose $f$ values are lower than $29 \mathrm{GHz}$ do not propagate through the $\beta$ region, which acts as a filter that prevents lower frequency SWs from entering into the Py $\gamma$ region after the Fe filter. On the other hand, those SWs with $f>29 \mathrm{GHz}$ propagate well

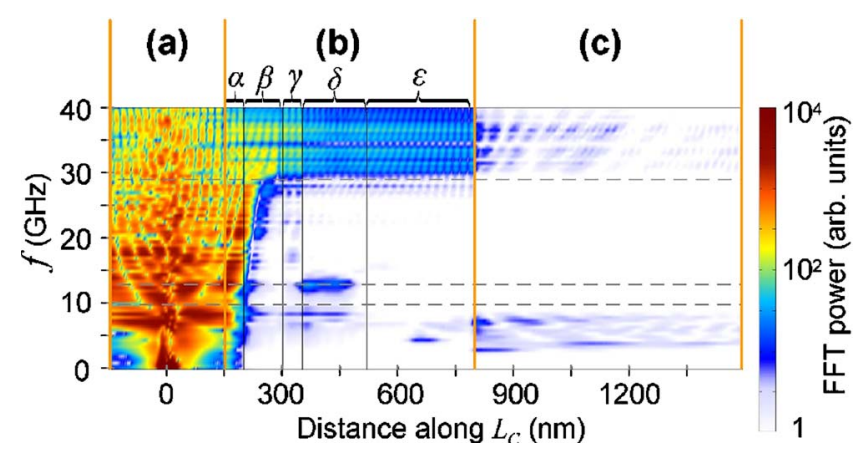

FIG. 4. (Color online) Frequency spectra along the black curved line noted as $L_{c}$, as shown in Fig. 1, which are obtained from the FFT of the temporal variations of the $M_{z} / M_{s}$ values. (a), (b), and (c) represent the individual regions of the circular disk, the Y-shaped waveguide, and the $\Xi$-shaped medium, respectively. The Y-shaped waveguide is classified into three regions, which correspond to a single channel $(\alpha, \beta$, and $\gamma)$, a curved channel $(\delta)$, and a branched channel $(\varepsilon)$ The Fe segment in the single channel is noted as $\beta$. The horizontal gray lines correspond to $f=10,13$, and $29 \mathrm{GHz}$. 


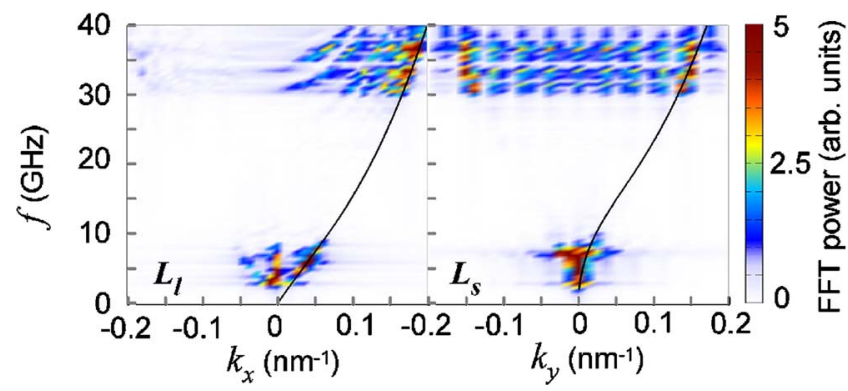

FIG. 5. (Color online) Dispersion relations calculated along the two different lines indicated by $L_{l}$ and $L_{s}$, as shown in Fig. 1 . The black-colored lines in the $+k$ domain are the results of theoretical calculations using an analytical dispersion equation reported in Ref. 12 for the cases where the propagation directions of SWs are parallel $\left(L_{l}\right)$ and perpendicular $\left(L_{s}\right)$ to the magnetization orientations.

through the Fe filter, then are transmitted into and travel through the entire length of the Y-shaped waveguide, before finally reaching $P_{2}{ }^{9}$. Thus, only those SWs of $f>29 \mathrm{GHz}$ are able to enter into the $\Xi$-shaped medium. Figure 4 thus visualizes how the radiation-mode SWs are injected from the magnetic vortex state into the magnetic nanowire and subsequently propagate through the Y-shaped waveguide and the E-shaped medium.

To better understand the characteristics of the propagation as well as interference in the $\Xi$-shaped medium, we calculate dispersion relations along the two different lines indicated by $L_{l}$ and $L_{s}$ (see Fig. 1), as shown in Fig. 5. In the two dispersions along the $L_{l}$ and $L_{s}$ lines, the lower and higher frequency regions are clearly divided by a forbidden region ranging from 10 to $29 \mathrm{GHz}$. The modes in the lower $f$ region are associated with the excitations of SWs in the $\Xi$-shaped medium itself. On the other hand, those in the higher $f$ region are associated originally with the radiationmode SWs that were once produced from the vortex core in the circular disk and originate directly from the transmission of the SWs with $f>29 \mathrm{GHz}$ through the Fe filter. Furthermore, those SWs in the higher region are quantized in the same manner as the quantization of the radiation modes in the circular disk, which are characterized by their various radial nodes. ${ }^{7,10-12}$

The clear differences in the dispersion relations between the $L_{s}$ and $L_{l}$ lines are related to the relative position of the lines with respect to the two diffraction sources of $P_{2}$. For the $L_{l}$ line, the two openings are lopsided toward the $-x$ direction of the $L_{l}$ line, so that most of the SWs propagate toward the $+x$ direction with respect to the $L_{l}$ line. Accordingly, those SW modes in the $+k$ domain have superior FFT powers to those in the $-k$ domain along the $L_{l}$ line. As regards the $L_{s}$ line, the two openings are positioned symmetrically along the $L_{s}$ line, so that those SWs propagating in both the $+y$ and $-y$ directions are equally dominating with respect to the $L_{s}$ line. Consequently, their FFT power distribution is symmetric with respect to the $f$ axis at $k_{y}=0$.

Another disparity is the shape of the mode distributions in the higher frequency region. The dispersion curves for the $L_{l}$ and $L_{s}$ lines vary with the relative orientation $\phi$ between the propagation direction and the orientation of the magneti- zations, as described by an analytical dispersion equation of $f=k(\phi)$ for a thin-film model with an infinite lateral dimension and a uniformly saturated magnetization. ${ }^{13}$ Due to the various directions of the propagation of the SWs diffracted from the two separate openings with respect to the individual lines of $L_{l}$ and $L_{s}$, various dispersion curves are to be expected depending on $\phi$, which is in accordance with the present simulation results. ${ }^{14}$ The black-colored curves are examples of the individual cases where $\phi=180^{\circ}$ for the $L_{l}$ line and $\phi=90^{\circ}$ for the $L_{s}$ line. ${ }^{15}$ This indicates that the wavelength of the SWs that are propagating along the direction perpendicular to the magnetization orientation is larger than that of the SWs that are propagating along the direction parallel to the magnetization orientation. We also note that the relative position of the two openings with respect to the $L_{s}$ and $L_{l}$ lines can determine the shape of the mode branches, such as the concave-up curves for the $L_{S}$ lines. ${ }^{16}$

To conclude, these presented results may offer a preview of the forthcoming era of magnetic logic devices using spin waves traveling at ultrafast speeds in nanowire-type magnetic waveguides.

This work was supported by the Korean Ministry of Science and Technology through the Creative Research Initiative Program.

${ }^{1}$ K.-S. Lee, S. Choi, and S.-K. Kim, Appl. Phys. Lett. 87, 192502 (2005). ${ }^{2}$ R. Hertel, W. Wulfhekel, and J. Kirschner, Phys. Rev. Lett. 93, 257202 (2004).

${ }^{3}$ T. Young, Philos. Trans. R. Soc. London 94, 1 (1804).

${ }^{4}$ We used the oommF code developed by M. J. Donahue and D. G. Porter (see http://math.nist.gov/oommf).

${ }^{5}$ The purpose of the thorny shape of the $\Xi$-shaped medium is to avoid the in-plane curling microstructures of the local magnetizations that are inevitably present in a confined micrometer-size magnetic element due to a long-range dipole-dipole interaction. Thus, SWs diffracted from the two openings marked by $P_{2}$ would travel well in the $\Xi$-shaped medium without being influenced by the inhomogeneous microstructures that would otherwise be present in such a finite-size confined medium.

${ }^{6}$ See EPAPS Document No. E-APPLAB-89-201632 for three movie files .mov. This Document can be reached via a direct link in the online article's HTML reference section or via the EPAPS homepage (http://www.aip.org/ pubservs/epaps.html).

${ }^{7}$ M. Buess, R. Höllinger, T. Haug, K. Perzlmaier, U. Krey, D. Pescia, M. R. Scheinfein, D. Weiss, and C. H. Back, Phys. Rev. Lett. 93, 077207 (2004). ${ }^{8}$ J. P. Park and P. A. Crowell, Phys. Rev. Lett. 95, 167201 (2005).

${ }^{9}$ The backward traveling waves, which are reflected from the individual boundaries situated between the circular disk, each of the $\alpha, \beta, \gamma, \delta$, and $\varepsilon$ regions, and the $\Xi$-shaped medium, produce a form of standing waves due to their superposition with the forward traveling waves, as is evidenced by the number of nodes with a length of $\lambda / 2$ along the propagation directions.

${ }^{10}$ K. Yu. Guslienko, W. Scholz, R. W. Chantrell, and V. Novosad, Phys. Rev. B 71, 144407 (2005).

${ }^{11}$ R. Zivieri and F. Nizzoli, Phys. Rev. B 71, 014411 (2005).

${ }^{12}$ B. A. Ivanov and C. E. Zaspel, Phys. Rev. Lett. 94, 027205 (2005).

${ }^{13}$ B. A. Kalinikos and A. N. Slavin, J. Phys. C 19, 7013 (1986).

${ }^{14}$ Due to the various oblique incidences of the spin waves to the observation lines, $L_{l}$ and $L_{s}$, the individual modes appear to satisfy $f=k(\phi)|\cos \phi|$ at the $L_{l}$ line and $f=k(\phi)|\sin \phi|$ at the $L_{s}$ line.

${ }^{15}$ This dispersion formula is approximate or assumed for the case of unpinned surface spins and the lowest mode, as in Ref. 13.

${ }^{16}$ Due to the page limit of this journal, the details of the mode branches will be reported elsewhere, along with the underlying physics of the discrete modes found along $k_{y}$ for the $L_{s}$ line. 